\title{
ON THE EXISTENCE OF DENSE ANALYTIC SUBGROUPS
}

\author{
DAVID ZERLING
}

\begin{abstract}
The purpose of this paper is to establish a necessary and sufficient condition for a connected Lie group to contain a proper dense connected Lie subgroup.
\end{abstract}

1. Introduction. By an analytic group and an analytic subgroup of a Lie group we mean a connected Lie group and a connected Lie subgroup, respectively. An analytic subgroup of $\operatorname{GL}(n, \mathbf{R})$ will be referred to as a linear analytic group. For an analytic group $G, Z(G)$ and $Z_{0}(G)$ will denote the center of $G$ and the identity component group of the center of $G$, respectively, and $\mathcal{L}(G)$ will denote the Lie algebra of $G$. $G^{\prime}$ will denote the commutator subgroup of $G$, so that, for example, if $K(G)$ is a maximal compact subgroup of $G$, then $K^{\prime}(G)$ will denote the commutator subgroup of $K(G)$.

The purpose of this paper is to establish a necessary and sufficient condition for an analytic group to contain a proper dense analytic subgroup. Several authors have elucidated the relationship between an analytic group and its dense analytic subgroups (cf. Djoković [1], Goto [2] and [3], and Zerling [6]) and in [5] Rajagopalan and Subrahmanian have developed a necessary and sufficient condition that a nondiscrete locally compact abelian group contain a proper dense subgroup.

\section{Main results.}

LEMMA 1. Let $G$ be an analytic group and let $N$ be a closed normal analytic subgroup of $G$ such that $G / N$ is a vector group $W$ of dimension at least one. Then $G=N \cdot V_{k} \cdot V_{k-1} \cdots V_{1}$, where $V_{i}, 1 \leqslant i \leqslant k$, are closed one dimensional vector subgroups of $G$ such that $N \cap V_{k}=N \cdot V_{k} \cdots V_{i+1} \cap V_{i}=$ $\{e\}, 1 \leqslant i \leqslant k-1$.

Proof. Let $\pi: G \rightarrow G / N$ be the natural projection. Let $W=W_{k}$. $W_{k-1} \cdots W_{1}$ be a direct product decomposition of $W$ into one dimensional vector subgroups. Then $\pi^{-1}\left(W_{i}\right)=N V_{i}$, where $V_{i}$ is a closed one dimensional vector subgroup of $G$ and $N \cap V_{i}=\{e\}$. Since $N$ is normal in $G$, we have $G=\pi^{-1}(W)=N \cdot V_{k} \cdot V_{k-1} \cdots V_{1}$. Since $N$ is the kernel of $\pi$ and since $W=W_{k} \cdot W_{k-1} \cdots W_{1}$ is a direct product decomposition, we see that $N \cap V_{k}=N \cdot V_{k} \cdots V_{i+1} \cap V_{i}=\{e\}, 1 \leqslant i \leqslant k-1$.

Received by the editors March 1, 1978.

AMS (MOS) subject classifications (1970). Primary 22E15.

Key words and phrases. Dense analytic subgroup, commutator subgroup, maximal compact subgroup.

๑ American Mathematical Society 1978 
LEMMA 2. Let $L$ be an analytic group and let $K(L)$ be a maximal compact subgroup of $L$. Suppose that $L^{\prime}$ is closed in $L$. Then the following statements are equivalent:

(a) $Z_{0}(K(L))$ contains a two dimensional toral subgroup whose intersection with $L^{\prime}$ is finite.

(b) $\operatorname{dim} K(L)-\operatorname{dim} K\left(L^{\prime}\right) \geqslant 2$, where $K\left(L^{\prime}\right)$ is a maximal compact subgroup of $L^{\prime}$.

Proof. Since $L^{\prime}$ is closed and normal in $L$, we see from Iwasawa [4] that $K(L) \cap L^{\prime}$ is a maximal compact subgroup of $L^{\prime}$, and since any two maximal compact subgroups of an analytic group are conjugate, we may assume that $K\left(L^{\prime}\right)=K(L) \cap L^{\prime}$. We have $K(L)=K^{\prime}(L) \cdot Z_{0}(K(L))$, where $K^{\prime}(L) \cap$ $Z_{0}(K(L))$ is finite, and

$$
\mathcal{L}(K(L))=\mathcal{L}\left(K^{\prime}(L)\right)+\mathcal{L}\left(Z_{0}(K(L))\right)
$$

is a direct sum. Since $K^{\prime}(L)$ is contained in $K\left(L^{\prime}\right)$, if we let $\mathcal{Q}$ denote the projection of $\mathcal{L}\left(K\left(L^{\prime}\right)\right)$ into $\mathcal{L}\left(Z_{0}(K(L))\right)$ we have the direct sum

$$
\mathcal{L}\left(K\left(L^{\prime}\right)\right)=\mathfrak{L}\left(K^{\prime}(L)\right)+\mathbb{Q} .
$$

Let $T_{1}$ denote the toral subgroup of $Z_{0}(K(L))$ generated by $\Theta$ and let $T$ be a toral subgroup of $Z_{0}(K(L))$ so that $Z_{0}(K(L))=T_{1} \cdot T, T_{1} \cap T=\{e\}$. Then $K\left(L^{\prime}\right)=K^{\prime}(L) \cdot T_{1}$, and $K(L)=K\left(L^{\prime}\right) \cdot T$, where $K\left(L^{\prime}\right) \cap T$ is finite. Therefore, $\operatorname{dim} K(L)-\operatorname{dim} K\left(L^{\prime}\right) \geqslant 2$ if and only if $\operatorname{dim} T \geqslant 2$ and our proof is now complete.

THEOREM. Let $L$ be an analytic group and let $K(L)$ be a maximal compact subgroup of $L$. Then in order that $L$ contain a proper dense analytic subgroup it is necessary and sufficient that one of the following conditions be satisfied:

(a) $L^{\prime}$ is closed in $L$ and $\operatorname{dim} K(L)-\operatorname{dim} K\left(L^{\prime}\right) \geqslant 2$, where $K\left(L^{\prime}\right)$ is a maximal compact subgroup of $L$.

(b) $L^{\prime}$ is not closed in $L$.

Proof. (i) To prove sufficiency let us first suppose that (a) is true. Then from Lemma 2 we have that $Z_{0}(K(L))$ contains a two dimensional toral subgroup whose intersection with $L^{\prime}$ is finite. Let $N$ be a maximal closed normal analytic subgroup of $L$ such that $N$ contains $L^{\prime}$ and such that $Z_{0}(K(L))$ contains a two dimensional toral subgroup whose intersection with $N$ is finite.

We have $K(L)=K^{\prime}(L) \cdot Z_{0}(K(L))$, where $K^{\prime}(L) \cap Z_{0}(K(L))$ is finite. Let $T_{1}$ denote the identity component group of $Z_{0}(K(L)) \cap N$. Then there is a toral subgroup $T$ of $Z_{0}(K(L))$ such that the dimension of $T$ is at least two and $Z_{0}(K(L))=T_{1} \cdot T, T_{1} \cap T=\{e\}$. We see that $N T$ is a closed normal analytic subgroup of $L$ containing both $K(L)$ and $L^{\prime}$, and $N \cap T$ is finite.

If $L=N T$, then letting $V$ be a dense one dimensional vector subgroup of $T$ we see that $G=N V$ is a proper dense analytic subgroup of $L$. We now show that $L=N T$. 
If $L \neq N T$, then since $N T$ contains $K(L)$ and $L^{\prime}$ we have that $L / N T$ is a vector group of dimension at least one. Then by Lemma 1 we have $L=$ $N T V_{k} \cdot V_{k-1} \cdots V_{1}$, where $V_{i}, 1 \leqslant i \leqslant k$, are closed one dimensional vector subgroups of $L$ such that $N T \cap V_{k}=N T V_{k} \cdots V_{i+1} \cap V_{i}=\{e\}, 1 \leqslant i \leqslant$ $k-1 . N V_{k}$ is a closed normal analytic subgroup of $L$ properly containing $N$. Also $N V_{k} \cap T$ is finite since $N T \cap V_{k}=\{e\}$ and $N \cap T$ is finite. This contradicts the maximality of $N$. Hence $L=N T$, and so $L$ contains a proper dense analytic subgroup.

Suppose now that (b) is true, that is, $L^{\prime}$ is not closed in $L$. Then from Goto [2, Proposition 14] we see that $K(L)$ is not contained in $L^{\prime}$. Let $N$ be a maximal analytic subgroup of $L$ containing $L^{\prime}$, but not containing $K(L)$.

As a first case suppose that $N \neq \bar{N}$. If $L \neq \bar{N}$, then since $\bar{N}$ contains $K(L)$ by the maximality of $N$ we see that $L / \bar{N}$ is a vector group of dimension at least one. Therefore, from Lemma 1 there exist closed one dimensional vector subgroups $V_{1}, V_{2}, V_{3}, \ldots, V_{k}$ of $L$ so that $L=\bar{N} \cdot V_{k} \cdot V_{k-1} \cdots V_{1}$, where $\bar{N} \cap V_{k}=N \cdot V_{k} \cdots V_{i+1} \cap V_{i}=\{e\}, 1 \leqslant i \leqslant k-1$. Then $N \cdot V_{k}$ properly contains $N$, but $N V_{k}$ does not contain $K(L)$, since $N$ is closed in $N V_{k}$ and $N$ is not closed in $L$. This contradicts the maximality of $N$. Therefore, $L=\bar{N}$, and $N$ is our desired proper dense analytic subgroup.

As a second case suppose $N=\bar{N}$. We have $K(L)=K^{\prime}(L) \cdot Z_{0}(K(L))$, where $K^{\prime}(L) \cap Z_{0}(K(L))$ is finite. Let $T_{1}$ denote the identity component group of $Z_{0}(K(L)) \cap N$. Since $K^{\prime}(L)$ is contained in $N$, but $K(L)$ is not contained in $N$, there is a toral group $T$ of dimension at least one so that $Z_{0}(K(L))=T_{1} \cdot T, T_{1} \cap T=\{e\}$, and $N T$ is a closed normal analytic subgroup of $L$ with $N \cap T$ finite. Since $N T$ contains both $L^{\prime}$ and $K(L)$ (by the maximality of $N), L / N T$ is a vector group. Using Lemma 1 and the maximality of $N$ we can see as before that $L=N T$. Furthermore, it is clear from the maximality of $N$ that the dimension of $T$ is exactly one.

Now let $K(N)=K(L) \cap N$. From Iwasawa [4] $K(N)$ is a maximal compact subgroup of $N$. $L^{\prime}$ is contained in $N$, but $L^{\prime}$ is not closed in $N$. Therefore, $L^{\prime}$ does not contain $K(N)$ by Goto [2]. Let $M$ be a maximal analytic subgroup of $N$ containing $L^{\prime}$, but not $K(N)$. If $M \neq \bar{M}$ we can show as before that $N / \bar{M}$ has dimension zero by the maximality of $M$, that is, $N=\bar{M}$ and $L=\bar{M} \cdot T$. Hence, $M T$ is our desired proper dense analytic subgroup of $L$. Therefore, we may suppose that $M=\bar{M}$.

We have $K(N)=K^{\prime}(N) \cdot Z_{0}(K(N))$, where $K^{\prime}(N) \cap Z_{0}(K(N))$ is finite. Let $T_{2}$ denote the identity component group of $Z_{0}(K(N)) \cap M$. Since $K^{\prime}(N)$ is contained in $M$, but $K(N)$ is not contained in $M$, there is a toral group $T_{3}$ of dimension one so that $Z_{0}(K(N))=T_{2} \cdot T_{3}, T_{2} \cap T_{3}=\{e\}$, and $M T_{3}$ is a closed normal analytic subgroup of $N$ with $M \cap T_{3}$ finite. Since $M T_{3}$ contains both $L^{\prime}$ and $K(N)$ (by the maximality of $M$ ), $N / M T_{3}$ is a vector group.

From Lemma 1 and the maximality of $M$ we can see as before that $N=M T_{3}$. Hence $L=N T=M T_{3} T$. Since $K(N)=K(L) \cap N$ and since $T$ 
is contained in $Z_{0}(K(L))$, we see that $T_{4}=T_{3} \cdot T$ is a two dimensional toral group and $L=M T_{4}$, where $M \cap T_{4}$ is finite. Letting $V$ be a dense vector subgroup of $T_{4}$ we see that $M V$ is a proper dense analytic subgroup of $L$, and $L=M \bar{V}=M T_{4}$, thus completing the second case, and, therefore, the sufficiency part of our proof.

(ii) To prove necessity let us suppose now that $L$ contains a proper dense analytic subgroup $G$. If $L^{\prime}$ is closed in $L$, then the following theorem of Goto [3] completes our proof. (We have altered the original statement of Goto's theorem, but a careful inspection of his proof justifies this alteration.)

Let $G$ be a dense analytic subgroup of an analytic group $L$ and suppose that $G$ contains a maximal normal analytic subgroup $N$ which contains the commutator subgroup of $G$ and is also closed in $L$. Then for each maximal compact subgroup $K$ of $L$ there exists a closed vector subgroup $V$ of $G$, such that $G=N V, N \cap V=\{e\}$, and $L=N \bar{V}$, where $N \cap \bar{V}$ is finite and $\bar{V}$ is a toral subgroup of $L$ which is central in $K$.

COROLlaRY 1. Let $L$ be a solvable linear analytic group. Then $L$ possesses $a$ proper dense analytic subgroup if and only if it contains a two dimensional toral subgroup.

Proof. The proof is a consequence of the above theorem and the wellknown fact that the commutator subgroup of a linear analytic group is closed and the commutator subgroup of a solvable linear analytic group is homeomorphic to Euclidean space.

COROllaRY 2. Let $L$ be a nilpotent linear analytic group. Then $L$ possesses a proper dense analytic subgroup if and only if $Z(L)$ contains a two dimensional toral subgroup.

Proof. The proof follows from Corollary 1 and the fact that any compact subgroup of $L$ will be contained in $Z(L)$, since the adjoint group of $L$ is homeomorphic to Euclidean space.

\section{Examples.}

EXAMPLE 1. Let $L=S L(2, \mathbf{R}) \times T$, where $T$ is a one dimensional toral group. Then $K(L)=S O(2) \times T$ is a maximal compact subgroup of $L$ which is a two dimensional toral group. But $K\left(L^{\prime}\right)=S O(2)$ is a maximal compact subgroup of $L^{\prime}$. Therefore, $L$ does not contain a proper dense analytic subgroup. We see that we cannot remove "solvable" from the hypothesis of Corollary 1.

EXAMPLE 2. Let $G$ denote the nilpotent analytic group of $3 \times 3$ matrices of the form

$$
\left(\begin{array}{ccc}
1 & a & c \\
0 & 1 & b \\
0 & 0 & 1
\end{array}\right) ; \quad a, b, c \in \mathbf{R} .
$$

$Z(G)$ consists of all matrices for which $a=b=0$. Let $H$ denote the discrete 
subgroup of $Z(G)$ consisting of all matrices for which $c$ is an integer. Let $L=G / H \times G / H$. Then $L$ is a nonlinear nilpotent analytic group in which $Z(L)=L^{\prime}=K(L)$, where $K(L)$, the maximal compact subgroup of $L$, is a two dimensional toral group. From our theorem $L$ does not contain a proper dense analytic subgroup. We see, therefore, that we cannot remove "linear" from either the hypothesis of Corollary 1 or the hypothesis of Corollary 2.

EXAMPLE 3. Let $L$ denote the solvable analytic group of matrices of the form

$$
\left[\begin{array}{ccc}
e^{2 \pi i r} & 0 & \alpha \\
0 & e^{2 \pi i s} & \beta \\
0 & 0 & 1
\end{array}\right) ; \quad r, s \in \mathbf{R}, \quad \alpha, \beta \in \mathbf{C} .
$$

Let $G$ denote the dense analytic subgroup of $L$ consisting of those matrices for which $s=\gamma r$, where $\gamma$ is a fixed irrational number. We see that $Z(L)=$ $\{e\}$. Therefore, we cannot remove "nilpotent" from the hypothesis of Corollary 2.

ACKNowledgement. The author would like to thank Professor Morikuni Goto for his constant encouragement and helpful discussions on Lie groups over the last several years.

\section{BIBLIOGRAPHY}

1. D. Ż. Djokovic, Three notes on real analytic groups, Tôhoku Math. J. (2) 28 (1976), 381-387.

2. M. Goto, Dense imbeddings of locally compact connected groups, Ann. of Math. (2) 61 (1955), 154-169.

3. __ Analytic subgroups of GL(n, R), Tôhoku Math. J. (2) 25 (1973), 197-199.

4. K. Iwasawa, On some types of topological groups, Ann. of Math. (2) 50 (1949), 507-558.

5. M. Rajagopalan and H. Subrahmanian, Dense subgroups of locally compact groups, Colloq. Math. 35 (1976), 289-292.

6. D. Zerling, Dense subgroups of Lie groups. II, Trans. Amer. Math. Soc. (to appear).

Department of Mathematics and Physics, Philadelphia College of Textiles and Science, Philadelphia, Pennsylvania 19144 